(A)

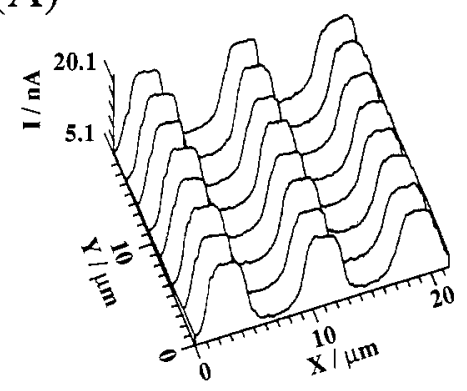

(B)

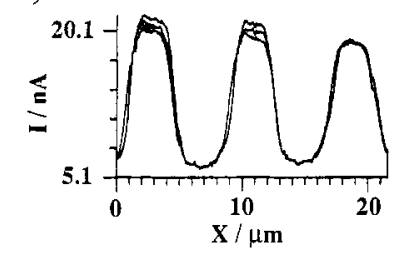

Figure 8. Scan of an IDA: tip, a method 1 electrode $(\sim 0.5-\mu \mathrm{m}$ diameter, a Pt hole in a $<0.3-\mu$ m-thick PMMA layer, see Figure 1D); $i_{T_{A}}=12.5 \mathrm{nA} ; E_{\mathrm{T}}=-0.77 \mathrm{~V}$ vs SCE; solution, same as in Figure 4B. (A) Three-dimensional view; (B) side view.

of these types could be equally useful in voltammetric studies and in STM experiments performed in situ in electrolyte solution. Their controllable sizes and predictable geometries are especially important in the SECM experiment. The implementation of mathmatical (e.g., deconvolution) schemes should improve the resolution of the SECM but would require electrodes with well-characterized shapes. While it is unlikely that the resolution of SECM will ever approach that of STM (atomic resolution), improvements to bring SECM to the level of several tens of nanometers, by reduction in tip size and by employing deconvolution or tomographic-type techniques, should be possible.

\section{ACKNOWLEDGMENT}

We acknowledge the assistance of I. Trachtenberg, M. Schmerling, and Daniel Mandler and the donation of the IDA by $O$. Niwa. This is paper number 6 in the SECM series.

\section{LITERATURE CITED}

(1) Bard, A. J.; Fan, F.-R. F.; Kwak, J. Lev. O. Anal. Chem. 1989, 61, 132.

(2) Bard, A. J.; Denuault, G.; Lee, C.; Mandler, D.; Wipt, D, O. AcC. Chem. Res ., in press.

(3) Kwak, J.; Bard, A. J. Anal. Chem. 1989, 61, 1221.

(4) Kwak, J.; Bard, A. J. Anal. Chem. 1989, 61, 1794.

(5) Wightman, R. M.; Wipf, D. O. In Electroanalytical Chemistry; Bard, A J., Ed.; Marcel Dekker: New York, 1989; Vol. 15, pp 267-353 and references therein.

(6) Pendley, B. D.; Abruña, H. D. Anal. Chem. 1990, 62, 782.

(7) Penner, R. M.; Heben, M. J.; Lewis, N. S. Anal. Chom. 1989, 61, 1630.

(8) Gewirth, A. A.: Craston, D. H.; Bard, A. J. J. Electroanal, Chem. Interfaclal Electrochem. 1989, $261,477$.

(9) Hepel, T.; Osteryoung, J. J. Electrochem. Soc. 1986, 133, 752.

(10) Hepel, T.; Osteryoung, J. J. Electrochem. Soc. 1986, 133, 7577.

(11) Aoki, K.; Morita, M.; Niwa, O.; Tabel, H. J. Electroanal. Chem. Interfacial Electrochem. 1988, 256, 269.

(12) Greeneich, J. S. In Electron Beam Technology in Microolectronic Fabrication; Brewer, G. R., Ed.; Academic Press: New York, 1980; Chapter 2.

RECEIVED for review July 9, 1990. Accepted September 28, 1990. The support of this research by NSF (CHE 8901450) and the Texas Advanced Research Program is gratefully acknowledged.

\title{
Vibrating Mirror Surface Plasmon Resonance Immunosensor
}

\author{
Rob P. H. Kooyman,* Aufried T. M. Lenferink, Rob G. Eenink, and Jan Greve \\ Department of Applied Physics, Twente University, P.O. Box 217, 7500 AE Enschede, The Netherlands
}

\section{INTRODUCTION}

In the past few years, much scientific and industrial effort has been put in the development of immunosensors (1). In these chemical sensors, antibodies are used as selector molecules to quantitatively determine the presence of a specified analyte, typically a protein. One class of optical immunosensors consists of devices where the optical evanescent field is exploited to probe changes at the interface between solution and antibody-covered substrate as a result of the binding between antibody and analyte.

The utilization of surface plasmon resonance (SPR) has been shown to be a promising method to be implemented in the development of these types of immunosensors (2-4). Generally, the angular-dependent reflectivity of light falling on a silver-coated prism onto which antibodies are immobilized provides information on the refractive index profile in the immediate vicinity $(\sim 100 \mathrm{~nm})$ of the interface, which can be interpreted in terms of the coverage of antibodies (2). As it is beyond the scope of the present paper, we will not discuss the theory of SPR (for a review, see ref 5); for our purposes, it suffices to mention that in the present context the main experimental parameter of a SPR experiment is the rate of

*Author to whom correspondence should be addressed. change of angular position of the reflectivity minimum. We will focus on the experimental determination of this parameter.

From previous experiments, it is known (2) that in order to measure protein concentrations of the order of $1 \mathrm{nM}$ one has to obtain an angular resolution of the order of $10^{-2} \mathrm{deg}$, given a plasmon resonance curve with a width of $2 \mathrm{deg}$. Several experimental approaches have been applied, such as monitoring the reflectivity in the region of maximum slope (3) and the use of photodiode arrays (6). A particularly elegant method was recently published, where use was made of an etched grating (4). This device, however, presently suffers from lack of sensitivity.

In the following, we will describe another method with properties that make it well suited for its use in the construction of SPR-based immunoassays.

\section{EXPERIMENTAL SECTION}

Figure 1a shows the optical arrangement. The sensor element consists of a glass microscope slide (Chance), coated with a silver layer of $\sim 50$-nm thickness, and optionally a polystyrene overlayer of about $20 \mathrm{~nm}(2)$.

Before the silver-coating procedure, the slides were cleaned by soaking in a detergent solution at $90^{\circ} \mathrm{C}$ in an ultrasonic bath for $\sim 30 \mathrm{~min}$ and subsequent rinsing in deionized water. 

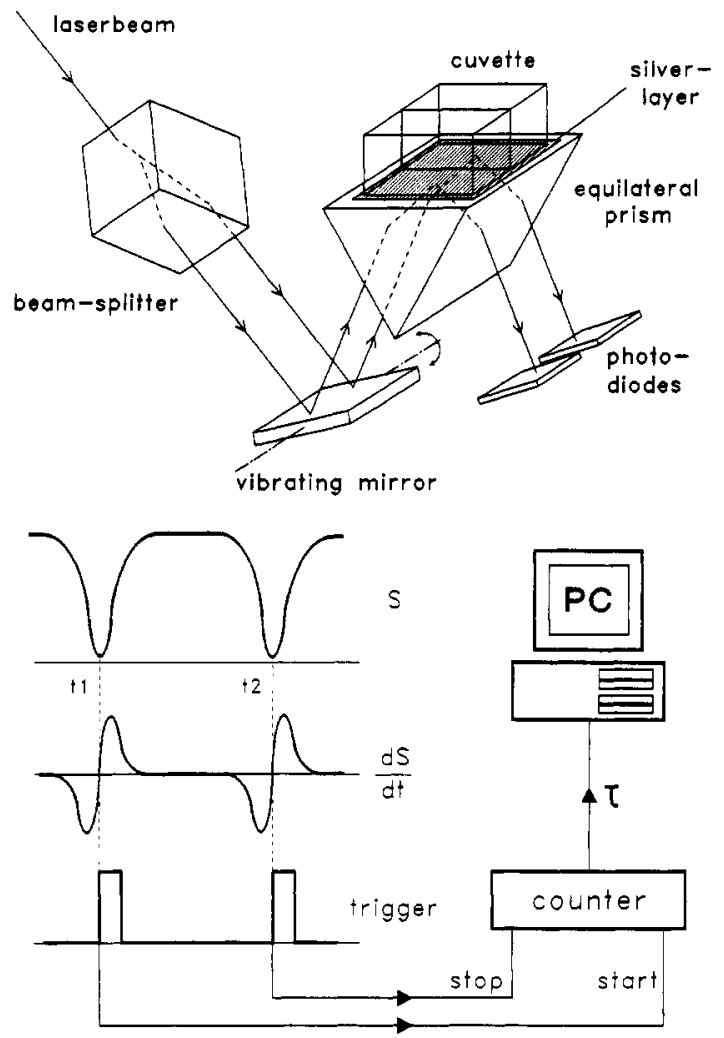

Figure 1. (a, top) Optical arrangement of the SPR immunosensor equipped with a vibrating mirror and two light paths to provide a detection and a reference channel. (b, bottom) Detection scheme for determining the plasmon angle. directly measured reflectance.

Antibodies are immobilized onto the silver or polystyrene overlayer. The protein solution to be quantified is applied as a drop onto the surface; alternatively, a small cuvette (ca. $150 \mu \mathrm{L}$ ) is glued onto the slide glass. This disposable unit is placed on top of an equilateral prism with refractive index identical with that of the slide glass. The optical contact between prism and slide glass is accomplished by a matching oil (Leitz).

Light from a $2-\mathrm{mW}$ HeNe laser (wavelength $633 \mathrm{~nm}$ ) is directed onto the prism surface by means of a vibrating mirror (Philips), intended for use in a video disk player; the intensity of the reflected light is monitored by a large-area photodiode (BPY63P). As shown in Figure 1a, the orientation of the cuvette permits an easy implementation of a reference system that can be used to compensate for unwanted responses (see Results and Discussion). For this purpose, the incoming light beam is split so that different analyte solutions can be monitored independently and simultaneously. Feeding the vibrating mirror by an ac current obtained from a function generator results in a device where the angle under which light strikes the surface has been made time-dependent.

The detection of the angle of minimum reflection $\theta_{\mathrm{p}}$ can be conveniently done on the basis of the principle as schematically indicated in Figure 1b. During one cycle of the vibrating mirror, the light traverses the reflectance minimum twice. At the instant $t_{1}$ at which the first minimum is traversed, a trigger pulse is generated to start an electronic counter; passage through the second minimum at $t_{2}$ provides a stop pulse. Thus, a changing angle $\left(\Delta \theta_{\mathrm{p}}\right)$ of minimum reflectance is transformed into a changing time span $(\tau)$ between start and stop pulses.

For amplitudes not too large (a few degrees from the equilibrium position), the angular deflection of the mirror is to a good approximation linear in a sinusoidal ac current

$$
\theta(t)=\theta_{\mathrm{m}} \sin (\omega t+\phi)
$$

where $\omega$ is the angular frequency of the mirror and $\theta_{m}$ is the angular amplitude.

As $\theta\left(t_{1}\right)=\theta\left(t_{2}\right) \equiv \theta_{\mathrm{p}}$, measurement of the quantity $t_{2}-t_{1} \equiv \tau$ provides a determination of $\theta_{\mathrm{p}}$, provided $\theta_{\mathrm{m}}$ is known:

$$
\theta_{\mathrm{p}}=\theta_{\mathrm{m}} \cos (\omega \tau / 2)
$$

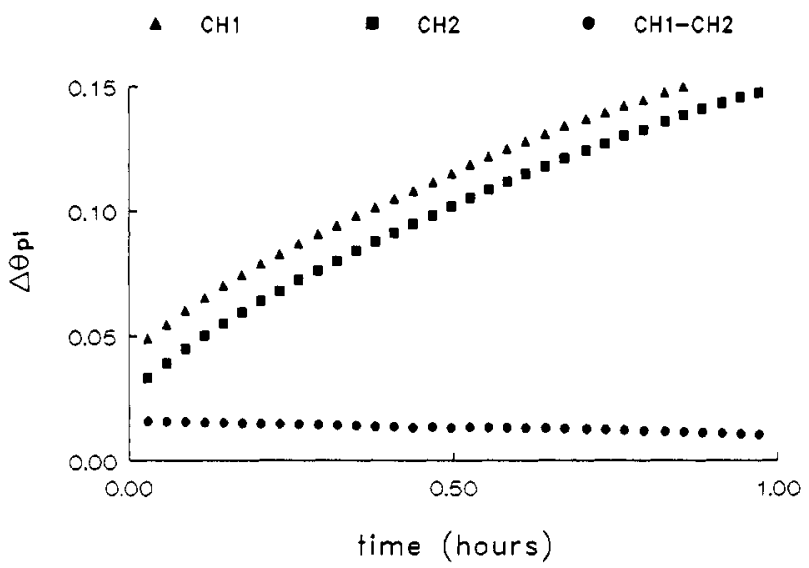

Figure 2. Drift correction using a reference channel (for the experiment, no antibodies were applied to the measured channel).

The instants $t_{1}$ and $t_{2}$ can be accurately measured by determining the zero points of the time derivative of the original signal. The parameter $\tau$ is processed online by a XT-type microcomputer equipped with a RTI- 800 interface board, to obtain a readout of $\theta_{\mathrm{p}}$ as a function of incubation time of the analyte.

\section{RESULTS AND DISCUSSION}

We have previously demonstrated that the dynamic range of this type of sensor is of the order of $10^{3}(7)$. This is mainly determined by the saturation behavior of the sigmoid curve of the binding isotherm near a coverage ratio of 1 . In the following, we will focus on the low concentration behavior of the device and we give some experimental results, illustrating its stability, angular resolution, and use of reference.

By electronic simulation of the start and stop signals, the total timing error in the electronic network, consisting of an analog differentiator and a counter, was found to be less than $1 \mu \mathrm{s}$, and thus, it is expected that the amount of jitter between the instantaneous value of the driver current and the spatial orientation of the mirror is the crucial parameter determining the overall angular stability of the system. When operating near the resonance frequency at ca. $60 \mathrm{~Hz}$, the jitter proved to be $\sim 5 \mu \mathrm{s}$, which we found quite remarkable for a mass product, such as this simple, inexpensive coil-operated mirror. If the time scale of the process of interest is larger than the sampling time of $\tau(\sim 20 \mathrm{~ms})$, the net jitter can be further reduced by averaging over a number of data points. In our application, where the characteristic time of the surface reaction is of the order of minutes, the net jitter was ca. $1 \mu \mathrm{s}$, obtained by averaging over 100 data points. In our setup, this corresponds to a measured angular stability of ca. $10^{-3} \mathrm{deg} / \mathrm{h}$.

However, if the device is used in a SPR setup to monitor, e.g., the immunoreaction, then it turns out that the main factor determining the net angular stability is the stability of the silver layer to chemical attack of the analyte solution. This is illustrated in Figure 2, where at $t=0$ the silver layer both in channel 1 and in channel 2 is brought in contact with the same buffer solution. It is seen that the reflectance minimum shifts over more than $0.1 \mathrm{deg}$, presumably because of adsorption processes. The difference signal, also shown in the figure, exhibits an improvement in the net stability of more than an order of magnitude.

In Figure 3 is shown how the obtained angular resolution makes it possible to measure very low surface coverages. Both of the surfaces in channels 1 and 2 are coated with an antibody layer. During the time interval $\mathrm{A}-\mathrm{B}$, the stirred solution in channel 1 is replaced by buffer, whereas in channel 2 a concentration $0.7 \mathrm{nM}$ protein (MW $\sim 4 \times 10^{4}$ ) in the same buffer is applied (B). It is seen that in both channels the angle of reflectance minimum undergoes a stepwise change (see Figure $3 a$ ); in channel 2 , a gradual angle change is superimposed, in 

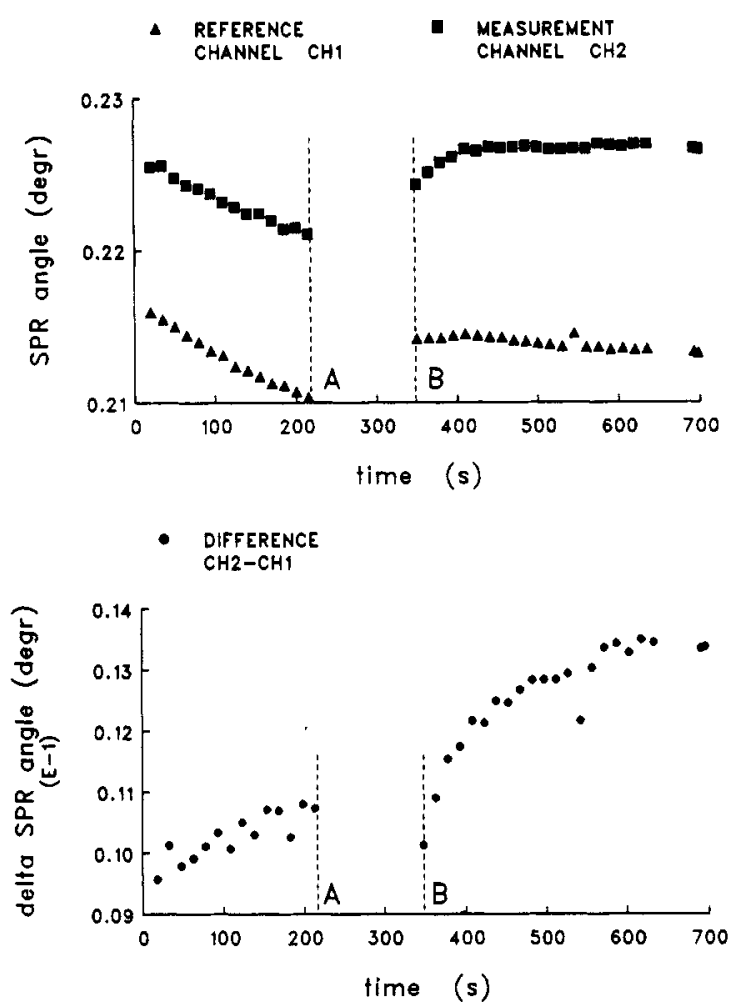

Figure 3. (a, top) SPR response of channel 1 and channel 2. First both channels contain only buffer. During the A-B interval, the solutions in both channels are replaced as indicated in the text. From $B$, immunoreaction takes place (only channel 2 ). (b, bottom) SPR response of the difference signal (channel 2 - channel 1).

this case indicative of a diffusion-controlled surface reaction (8). The net effect is an angle shift of about $4 \times 10^{-3} \mathrm{deg}$ (see Figure $3 \mathrm{~b}$ ), corresponding to a covered fraction of $\sim 0.01$. Furthermore, from the figure, the overall angular resolution can be estimated at $2 \times 10^{-3} \mathrm{deg}$.

\section{CONCLUSIONS}

We have described a simple and inexpensive device to determine a reflectance minimum. Although its use was demonstrated in a SPR setup, it is not restricted to this technique.
The vibrating mirror device has a number of advantages over previously published setups: because the device allows for time averaging, it has superior angular resolution compared to a rotation stage $\left(10^{-3} \mathrm{vs} 10^{-2} \mathrm{deg}\right)$; furthermore, the response is essentially independent of slow fluctuations in ambient light and/or laser intensity, as the detected parameter is a minimum in light intensity. Another useful feature is that the device has a relatively high dynamic response ( $\sim 50 \mathrm{~ms})$, combined with an angular sensitivity of ca. $0.01 \mathrm{deg}$. The response time is primarily determined by the vibration frequency of the mirror. The complete reflectance curve can thus be recorded within this time interval, which allows for the study of relatively fast surface processes. In this context, it can be considered as an additional advantage that a true minimum of the reflectance is measured, in contrast to turntable devices, where, in order to obtain sufficient sensitivity, the angle $\theta$ is kept fixed in the region of maximum slope (4). Finally, from a practical point of view it is not unimportant that the device is inexpensive.

A disadvantage of the present setup is that during an angular scan the probed spot is not stationary and can shift $0.1-1$ $\mathrm{mm}$ over the surface. For SPR experiments on surfaces that are inhomogeneous over this characteristic length, this can complicate the interpretation of the experimental results. An alternative setup is under development, where the spot position remains stationary.

The use of a reference channel has been shown to considerably improve the overall angular stability. Apart from its application in compensation for unwanted silver responses (e.g., temperature, nonspecific adsorption), the reference channel is useful in minimizing other common channel effects such as variations in the composition of the analyte solution.

\section{LITERATURE CITED}

(1) Nylander, C. J. Phys. E: Sci Instrum 1985, $18,736$.

(2) Kooyman, R. P. H.; Kolkman, H.; Gent, J. van; Greve, J. Anal. Chim Acta 1988, 35, 213.

(3) Liedberg, B.; Nylander, C.; Lundstrom, I. Sens. Actuators 1983, 4 299.

(4) Cullen, D. C.; Brown, R. G. W.; Lowe, C. R. Biosensors 1988, 3, 211

(5) Raether, H. In Physics of Thin Films; Hass, G. Francombe, M., Hoffman, R., Eds.; Academic Press: New York, 1977; Vol. 9.

(6) Matsubara, K.; Kawata, S.; Minami, S. Appl. Opt. 1988, 27, 1160.

(7) Kooyman, R. P. H.; de Bruijn, H. E.; Eenink, R. G.; Greve, J. J. Mol. Struct. 1990, 218. 345.

(8) Eddowes, M. J. Biosensors 1988, 3, 1.

\title{
Appllcation of (3-Mercaptopropyl)trimethoxysilane as a Molecular Adhesive in the Fabrication of Vapor-Deposited Gold Electrodes on Glass Substrates
}

\author{
Charles A. Goss, Deborah H. Charych, and Marcin Majda* \\ Department of Chemistry, University of California, Berkeley, California 94720
}

\section{INTRODUCTION}

Vapor deposition of $50-20$-nm gold films on glass and $\mathrm{SiO}_{2}$ substrates have been used as a convenient and consistently reproducible method of electrode fabrication $(1-10)$ and in the preparations of standard gold substrates for self-assembly of alkylthiol monolayers and their structural studies (11-17). In electrochemistry, this method of electrode fabrication has been particularly successful in experiments involving electrode modifications by spin coating of polymer films $(1,6)$ and silanization reactions (2) and by formation of Langmuir-
Blodgett and self-assembling films of amphiphiles $(3,7,10)$. In these types of experiments, the availability of a large number of optically flat, disposable electrodes is a necessity. Vacuum deposition techniques have been also used in the fabrication of interdigitated microelectrode arrays $(18,19)$ and electrochemical devices (20).

In all these applications, ca. 5-nm transition-metal underlayers have to be vapor-deposited first to enhance the gold adhesion to the substrate $(1-4,11-18,20)$. Otherwise, directly deposited gold films do not withstand chemical treatment such 